\title{
Hyperinsulinämie tangiert Vagus nicht
}

Fragestellung: Wie beeinflusst eine schrittweise Erhöhung der Serum-Insulinkonzentration die kardiale Vagusfunktion?

Hintergrund: Menschen mit Übergewicht und Insulinresistenz haben eine geringere Herzfrequenzvariabilität (HFV) als Gesunde. Dies ist assoziiert mit einer erhöhten allgemeinen und kardialen Mortalität bei kardiologischen Patienten. Einer der möglichen Gründe für eine erniedrigte HFV ist die Abnahme der kardialen Vaguskontrolle. Bei Patienten mit Insulinresistenz, meist begleitet vom metabolischem Syndrom, sind hohe Insulinkonzentrationen ein möglicher Grund. Viele Studien ergaben, dass ein kurzfristiger Anstieg von Insulin die HFV verringert und/oder die Herzfrequenz (HF) ansteigen lässt. In den meisten Studien wurde der Effekt des N. sympathicus auf das Herz geprüft, nur wenige haben bisher den Zusammenhang zwischen Insulin und Vaguskontrolle erforscht. Die atemabhängige HF-Variabilität ist die beste Methode, um die kardiale Vaguskontrolle zu untersuchen. Bei der Fragestellung der Studie interessierte besonders, welchen Effekt GLP-1 zusätzlich auf die Vagusfunktion hat. Getestet wurde, ob höhere Insulinwerte mit einer geringeren kardialen vagalen Kontrolle einhergehen, und zwar unabhängig von BMI und der Insulinresistenz und, ob ein experimentell induzierter Anstieg von exogenem oder endogenem Insulin die kardiale Vagusfunktion negativ beeinflusst.

Patienten und Methodik: EKG und respiratorische Funktionen wurden bei 130 gesunden Teilnehmern im euglykämisch-hyperinsulinämischen Clamp
Berkelaar M, Eekhoff EM, Simonis-Bik AM et al. Effects of induced hyperinsulinaemia with and without hyperglycaemia on measures of cardiac vagal control. Diabetologia 2013; 56(6):1436-43. untersucht, ergänzt durch einen modifizierten hyperglykämischen Clamp mit GLP-1 und Arginin sowie Substanzen, die zu einer endogenen Stimulation von Insulin führen. Variablen kardialer Vaguseffekte wurden in Relati-

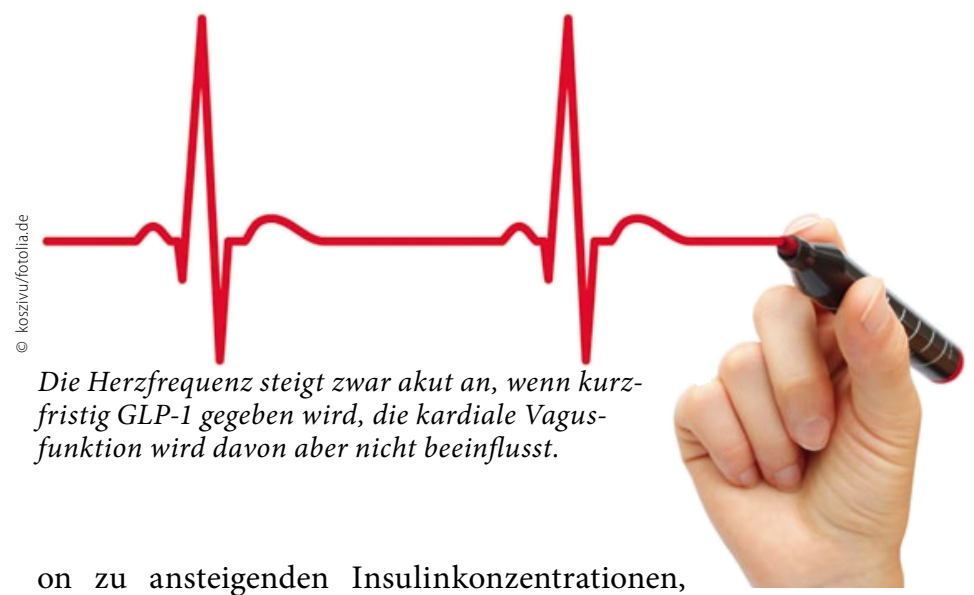

GLP-1 und GLP-1 und Arginin untersucht (HFV,

HF, rMSSD [root mean square of successive differences], pvRSA [peak valley respiratory sinus arrhythmia], HF [high frequency power]).

Ergebnisse: Höhere Insulinspiegel gingen mit höherer HF und verminderter RSA einher, außer im euglykämischen Clamp. Insulin war negativ assoziiert mit den Variablen vagaler Parameter, auf Signifikanzniveau für rMSSD und $\log 10$ HF. Nach Adjustierung (Alter, BMI, Insulinsensitivität) schwanden die Korrelationen. GLP-1 und Arginin ließen die HF signifikant steigen, was aber nicht mit signifikanter HFV-Reduktion gekoppelt war.

Schlussfolgerungen: Eine experimentell induzierte Hyperinsulinämie korreliert nicht mit der kardialen Vaguskontrolle oder der HF, wenn man Alter, BMI und Insulinsensitivität adjustiert. Eine endogene Insulinstimulation mit GLP-1 während einer Hyperglykämie lässt die HF akut ansteigen, mindert aber die kardiale vagale Kontrolle nicht. Ob eine Langzeit-Therapie mit GLP-1 bei Diabetikern die kardiale vagale Kontrolle langfristig reduzieren kann, sollte weiter in Studien untersucht werden.

stieg signifikant an. Ob das bei Diabetikern eine Rolle spielt, oder ob eine Langzeittherapie mit GLP-1 die kardiale Vagusfunktion negativ beeinflussen könnte, muss man, wie von den Studienleitern gefordert, weiter untersuchen.

Die Daten zeigen, dass BMI und Insulinresistenz die entscheidenden Determinanten der Herzfrequenz (HF) und der kardialen vagalen Kontrolle sind und nicht der Insulinspiegel selbst, zumindest bei experimentell induzierter Hyperinsulinämie. Für die Praxis heißt das, dass eine Insulintherapie per se die vagale Herzfunktion nicht negativ zu beeinflussen scheint und, dass für die kardiale Vagusfunktion die Insulinresistenz und das Körpergewicht wichtiger zu sein scheint. Auch für die endogene Insulinstimulation mit GLP-1 konnte bei gesunden Probanden bei Hyperglykämie kein signifikant negativer Einfluss auf die kardiale vagale Funktion gezeigt werden, aber die HF

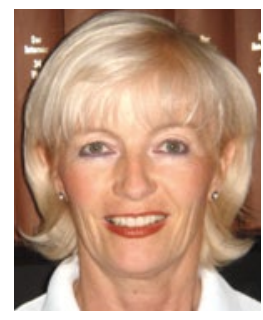

Dr. med. Cornelia Jaursch-Hancke

Schwerpunkt Endokrinologie, Diabetes und Stoffwechsel

Stiftung Deutsche Klinik für Diagnostik GmbH Aukammallee 33, 65191 Wiesbaden cornelia.jaursch-hancke@dkd-wiesbaden.de 